\title{
SOFIA observations of S106: dynamics of the warm gas $\star$
}

\author{
R. Simon ${ }^{1}$, N. Schneider ${ }^{2}$, J. Stutzki ${ }^{1}$, R. Güsten ${ }^{3}$, U. U. Graf ${ }^{1}$, P. Hartogh ${ }^{4}$, \\ X. Guan ${ }^{1}$, J. G. Staguhn ${ }^{5,6}$, and D. J. Benford ${ }^{5}$ \\ 1 KOSMA, I. Physikalisches Institut, Universität zu Köln, Zülpicher Str. 77, 50937 Köln, Germany \\ e-mail: simonr@ph1.uni-koeln.de \\ ${ }^{2}$ Laboratoire AIM Paris Saclay, CEA/Irfu - Université Paris Diderot - CNRS, Centre d'Études de Saclay, 91191 Gif-sur-Yvette, \\ France \\ e-mail: nicola.schneider-bontemps@cea.fr \\ 3 Max-Planck Institut für Radioastronomie, Auf dem Hügel 69, 53121 Bonn, Germany \\ 4 Max-Planck-Institut für Sonnensystemforschung, Max-Planck-Str. 2, 37191 Katlenburg-Lindau, Germany \\ 5 Observational Cosmology Laboratory (Code 665), NASA Goddard Space Flight Center, Greenbelt, MD 20771, USA \\ ${ }^{6}$ Department of Physics \& Astronomy, Johns Hopkins University, Baltimore, MD 21218, USA \\ Received 31 January 2012 / Accepted 14 March 2012
}

\section{ABSTRACT}

\begin{abstract}
Context. The H II region/PDR/molecular cloud complex S106 is excited by a single O-star. The full extent of the warm and dense gas close to the star has not been mapped in spectrally resolved high- $J$ CO or [C II] lines, so the kinematics of the warm, partially ionized gas, are unknown. Whether the prominent dark lane bisecting the hourglass-shaped nebula is due solely to the shadow cast by a small disk around the exciting star or also to extinction in high column foreground gas was an open question until now.

Aims. We disentangle the morphology and kinematics of warm neutral and ionized gas close to the star, study their relation to the bulk of the molecular gas, and we investigate the nature of the dark lane.

Methods. We used the heterodyne receiver GREAT onboard on SOFIA to observe velocity resolved spectral lines of [C II] and $\mathrm{CO} 11 \rightarrow 10$ in comparison with so far unpublished submm continuum data at $350 \mu \mathrm{m}$ (SHARC-II) and complementary molecular line data.

Results. The high angular and spectral resolution observations show a very complex morphology and kinematics of the inner S106 region, with many different components at different excitation conditions contributing to the observed emission. The [C II] lines are found to be bright and very broad, tracing high velocity gas close to the interface of molecular cloud and $\mathrm{H}$ II region. CO $11 \rightarrow 10 \mathrm{emis}-$ sion is more confined, both spatially and in velocity, to the immediate surroundings of S106 IR showing the presence of warm, high density (clumpy) gas. Our high angular resolution submm continuum observations rule out the scenario where the dark lane separating the two lobes is due solely to the shadow cast by a small disk close to the star. The lane is clearly seen also as warm, high column density gas at the boundary of the molecular cloud and $\mathrm{H}$ II region.
\end{abstract}

Key words. HII regions - ISM: individual objects: S106 - ISM: kinematics and dynamics - photon-dominated region (PDR) ISM: clouds

\section{Introduction}

The $\mathrm{H}$ II region $\mathrm{S} 106$ at a distance of 1.2 to $1.8 \mathrm{kpc}$ (Schneider et al. 2007) in Cygnus is a prominent bipolar emission nebula associated with an extended molecular cloud. The nebula is excited by the single, late O-type star S106 IR, which creates a bright photon-dominated region (PDR) at the molecular cloud interfaces. In addition, S106 IR drives an ionized wind with a velocity of $\sim 200 \mathrm{~km} \mathrm{~s}^{-1}$ (Simon \& Fischer 1982) that is responsible for the hourglass shape and high velocity wings seen in optically thick molecular line emission close to the star. Here, we focus on the immediate surroundings of S106 IR, relevant for the context of the SOFIA observations. More details and references are given in Hodapp \& Schneider (2008) and by Schneider et al. (2002, 2003, 2007).

The two lobes of ionized gas seen in the optical and radio continuum are separated by a dark lane that is very prominent in near- to mid-IR imaging (Smith et al. 2001; Oasa et al. 2006). (Sub)Millimeter dust and optically thin molecular line emission

\footnotetext{
* Appendix $\mathrm{A}$ is available in electronic form at http://www . aanda.org
}

(Richer et al. 1993; Schneider et al. 2002, 2003) show two emission peaks $\sim 15^{\prime \prime}$ east and west of S106 IR in the dark lane, while the bulk of the molecular gas is located further to the east and west of the lobes. Two clusters of $\mathrm{H}_{2} \mathrm{O}$ masers were detected at the western peak (S106 FIR, Stutzki et al. 1982; Furuya et al. 1999). Richer et al. (1993) interpret this source as a Class 0 young stellar object.

While the dark lane was initially interpreted as a smooth, large-scale disk (Bally \& Scoville 1982; Little et al. 1995), Barsony et al. (1989) and Richer et al. (1993) concluded that the molecular line and dust continuum emission arises from a clumpy molecular cloud, possibly the dense remnants of a large disk or torus disrupted by the central star. A high degree of clumpiness was already inferred early on through observations of ammonia (Stutzki \& Winnewisser 1985). Whether the dark lane is just a shadow cast by a small, edge-on (accretion) disk close to the star (Bally et al. 1983; Noel et al. 2005), largely confining the ionizing radiation to the optical/radio lobes, or in addition due to dust extinction in high column density gas, is a matter of ongoing debate. A possible detection of the disk was reported by Hoare \& Muxlow (1996) and Gibb \& Hoare (2007). 
Observations in mid- to high- $J$ CO lines, specifically tracing PDR gas close to S106 IR, indicate the presence of warm $(T>200 \mathrm{~K})$ and dense $\left(n>10^{5} \mathrm{~cm}^{-3}\right)$ gas (Harris et al. 1987; Graf et al. 1993; Richer et al. 1993; Little et al. 1995; Schneider et al. 2003). Schneider et al. (2003) observed a larger area (few arcmin) in [C II] $158 \mu \mathrm{m}$ using the Kuiper Airborne Observatory (KAO), and submm [C $\mathrm{I}]$ and CO lines (KOSMA $3 \mathrm{~m}$ ), revealing the whole spatial extent of the PDR region. Higher angular resolution [O I] observations towards the center provide a more detailed view of the higher density PDR gas. The [C II] and $\left[\mathrm{O}_{\mathrm{I}}\right] \mathrm{KAO}$ data, however, were not velocity resolved.

\section{Observations}

The [C II] atomic fine structure line at $1.900537 \mathrm{THz}$ $(157.737 \mu \mathrm{m})$ and the $\mathrm{CO} 11 \rightarrow 10$ rotational line at $1.267 \mathrm{THz}$ were observed with the heterodyne receiver GREAT ${ }^{1}$ (German REceiver for Astronomy at Terahertz frequencies) onboard SOFIA during two flights on April 13 and July 22, 2011, from Palmdale/California. Total power on-the-fly maps with a scanning speed of $8^{\prime \prime} / \mathrm{s}, 1 \mathrm{~s}$ dump time, and a typical size of $2^{\prime} \times 2^{\prime}$ were performed. Positional offsets refer to the star S106 IR (RA, Dec $)(\mathbf{J} 2000)=\left(20^{\mathrm{h}} 27^{\mathrm{m}} 26^{\mathrm{s}} .74,37^{\circ} 22^{\prime} 47^{\prime \prime}\right.$ '9). The region centered on S106 IR has three coverages while the western extension has only one to two coverages, resulting in a non-uniform noise distribution. Blank sky subtraction was done towards a position offset by $\left(8^{\prime}, 0^{\prime}\right)$, well outside any emission seen in the KAO [C II] map presented by Schneider et al. (2003). Instrument alignment and telescope efficiencies, antenna temperature and atmospheric calibration, as well as the spectrometers used are described in Heyminck et al. (2012) and Guan et al. (2012). Here, we only show data from one of the Fast Fourier Transform Spectrometers (AFFTS), the other spectrometers giving redundant information. All line intensities are reported as main beam temperatures scaled with main-beam efficiencies of 0.51 and 0.54 for $\left[\mathrm{C}_{\mathrm{II}}\right]$ and $\mathrm{CO}$, and a forward efficiency of $95 \%$. The mean r.m.s. noise temperatures per $0.5 \mathrm{~km} \mathrm{~s}^{-1}$ velocity bin for the central region (western extension) are 2.2 (3.7) $\mathrm{K}$ for $[\mathrm{C} \mathrm{II}]$ (16" beam) and 1.8 (2.4) K for CO (19.6" beam). The absolute calibration uncertainty is estimated to be $\sim 10 \%$. The IRAM $30 \mathrm{~m}$ observations used for comparison with the new SOFIA data are described in Schneider et al. (2002).

The $350 \mu \mathrm{m}$ continuum observations were obtained in March, 2004, with the SHARC-II bolometer camera at the $\mathrm{CSO}^{2}$. Our scanning strategy was to modulate the telescope pointing with a non-connecting Lissajous pattern within the limits of reasonable telescope acceleration (typical periods of $20 \mathrm{~s})$. Calibration was frequently verified on planets and moons. Pointing was checked about every hour on evolved stars and blazars. Intensities are given in Jy/beam with a beam size of $9^{\prime \prime}$.

\section{Results and discussion}

\subsection{Global morphology and the dark lane}

Figure 1 shows the integrated intensity distribution of [C II] $158 \mu \mathrm{m}$ and CO $11 \rightarrow 10$ emission as observed with SOFIA. The emission morphology in both lines shows a pronounced peak centered on S106 IR and extended emission towards the south,

\footnotetext{
${ }^{1}$ GREAT is a development by the MPI für Radioastronomie and the KOSMA/Universität zu Köln, in cooperation with the MPI für Sonnensystemforschung and the DLR Institut für Planetenforschung.

2 The Caltech Submillimeter Observatory is operated by the California Institute of Technology under cooperative agreement with the National Science Foundation (AST-0838261).
}

north-west, and north-east, creating the heart-shaped emission already seen in the KAO data. The $\mathrm{CO}$ emission is more confined to the central region, offset from the emission peaks seen in $[\mathrm{C}$ II], and better matches the [O I] emission from the dense PDR gas (Schneider et al. 2003). The SHARC-II $350 \mu \mathrm{m}$ data show S106 FIR as a bright, unresolved source west of S106 IR, while east of the star, the emission is elongated to the north-east with some fainter extension towards the south-east resembling a dusty cone. Close to S106 IR, the integrated [C II] intensity has two lobes perpendicular to the submm continuum emission.

Near-IR and cm continuum show the northern and southern lobe divided by the dark cone in the east. Near S106 IR, the two [C II] peaks closely follow the $\mathrm{cm}$ continuum, suggesting that part of the $[\mathrm{C} \mathrm{II}]$ emission is coming from the $\mathrm{H}_{\mathrm{II}}$ region, while the more extended $\left[\mathrm{C}_{\mathrm{II}}\right]$ is unrelated to the $\mathrm{cm}$ continuum. The Subaru image does not trace the full extent of the H II region just north- and south-east of S106 IR. Here, there is clearly radio continuum emission from behind the dark cone in the foreground. The submm continuum emission perfectly matches the dark lane as well as the fainter near-IR dark regions just south of it. These observations reveal that the dark lane and cone are not just a shadow of the small disk around S106 IR, but indeed high column density, warm gas at the edge of the molecular cloud (traced by the $-2 \mathrm{~km} \mathrm{~s}^{-1}$ channel of ${ }^{13} \mathrm{CO} 2 \rightarrow 1$ in Fig. A.1), located in front of the $\mathrm{H}$ in region. The shadow of the small central disk protects the dark lane from ionizing UV radiation and likely is responsible for the fact that the lane survived for a significant time of the nebula's evolution after the formation of the central star. We will now use our new SOFIA and complementary IRAM $30 \mathrm{~m}$ spectral line data to study the kinematics of the warm gas in the context of the nebula structure and geometry. For this purpose, we include various channel map overlays in the Appendix that reveal how complex the emission in the different velocity components and for the different tracers is. We, therefore, focus this letter on the analysis and discussion of the morphology and kinematics of S106 and defer a more quantitative analysis, including PDR modeling, to a later paper.

\subsection{Velocity structure}

Channel maps of $[\mathrm{C}$ II $]$ and $\mathrm{CO} 11 \rightarrow 10$ emission (top panel of Fig. A.1) reveal the different velocity components of warm and dense gas in S106. The emission can be divided into three major ranges for $[\mathrm{C} \mathrm{II}]$ : (1) localized blue emission close to S106 IR $\left(v=-6\right.$ to $\left.-3 \mathrm{~km} \mathrm{~s}^{-1}\right)$, (2) extended emission with the characteristic heart-shape and an extension to the south at RA offset $-70^{\prime \prime}$ associated with the velocity of the bulk of molecular gas $\left(v=-3\right.$ to $1 \mathrm{~km} \mathrm{~s}^{-1}$, see low- $J$ CO spectra in Fig. 2 and channel maps in Fig. A.1), (3) and faint red emission spatially coinciding with the southern $\mathrm{H}_{\text {II }}$ region lobe $(v=0$ to $4 \mathrm{~km} \mathrm{~s}^{-1}$ and higher). CO $11 \rightarrow 10$ is offset from the [C II] emission in all channel maps, with $\left[\mathrm{C}_{\mathrm{II}}\right]$ often wrapping around the $\mathrm{CO}$. These differences are due to the different excitation conditions. CO $11 \rightarrow 10$ is prominent closer to the star as it traces the warmer, dense clumps mainly associated with the dark cone close to S106 IR (the $J=11$ level is $365 \mathrm{~K}$ above ground and the critical density is $>10^{6} \mathrm{~cm}^{-3}$ ), while [C II] traces PDR or shocked material at the interfaces of the cloud and the $\mathrm{H}$ II region.

The finding that the northern lobe is dominated by blue and the southern by red [C $\mathrm{CI}]$ emission is surprising as the southern lobe is tilted towards the observer while the northern lobe is pointing away (e.g., Solf \& Carsenty 1982). For a proper explanation, we need to consider what is known about the geometry and dynamics of the nebula. The lobes of the 

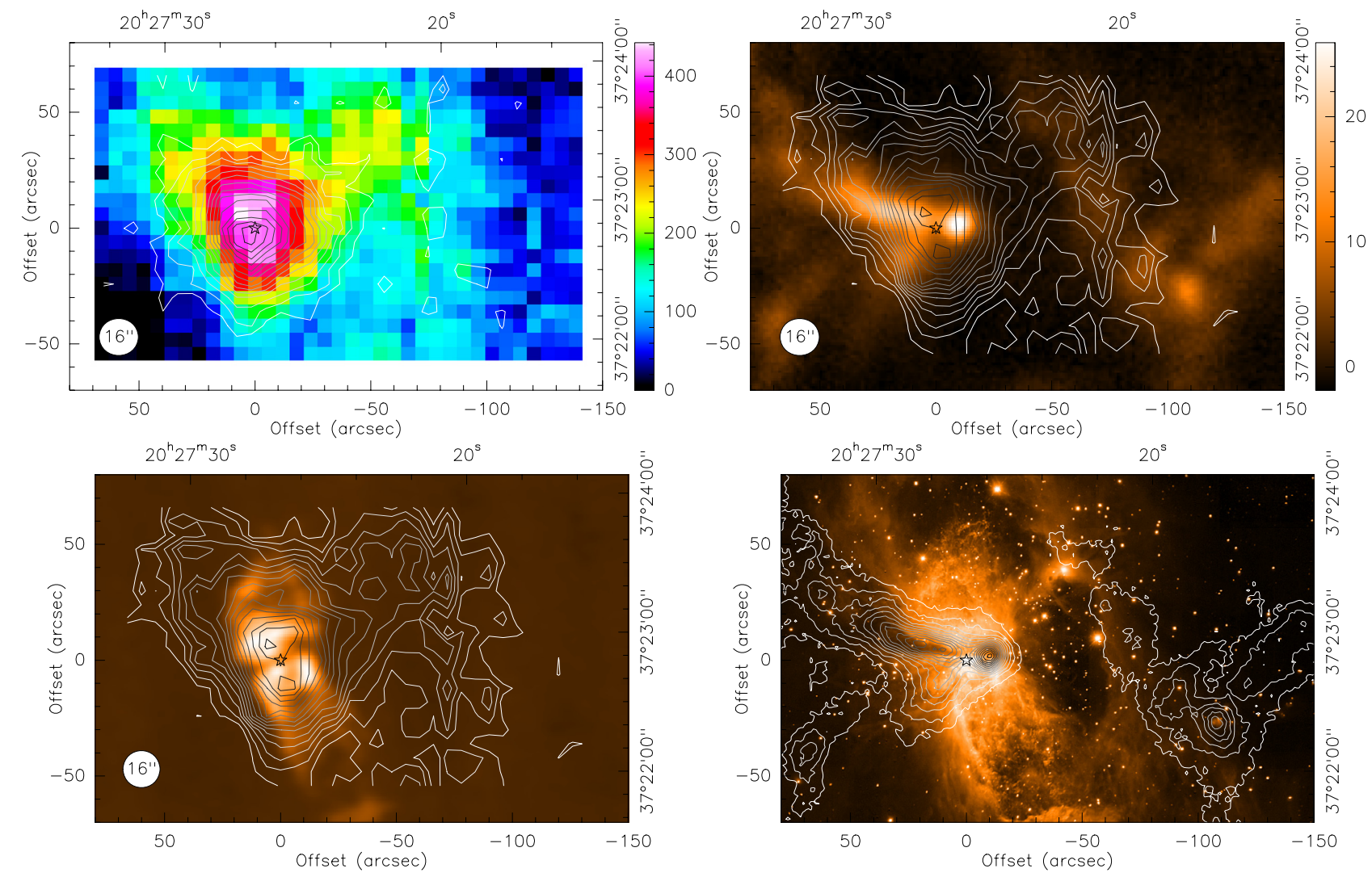

Fig. 1. Top: integrated intensity ( $v_{\mathrm{LSR}}-20$ to $20 \mathrm{~km} \mathrm{~s}^{-1}$ ) of [C II] (color) and CO $11 \rightarrow 10$ (contours from 15 to 215 in steps of $15 \mathrm{~K} \mathrm{~km} \mathrm{~s}$ s. $^{-1}$. The position of S106 IR is indicated by a star and the SOFIA beam size for [C II] is drawn in the bottom left corner. On the right, contours of [C II] integrated intensity are shown on the SHARC-II $350 \mu$ m image (intensities in Jy/beam). Bottom: overlays of [C II] on $1.47 \mathrm{GHz}$ radio continuum (left, VLA archival data) and SHARC-II $350 \mu \mathrm{m}$ contours (right) on the Subaru near-IR image.

hourglass nebula expand both radially and along the main axis (Hodapp \& Schneider 2008) driven by S106 IR. The wind and the expanding lobes are expected to sweep up material where they hit molecular gas, except for the front wall of the southern lobe, which has been partly eroded by the star (the back side of the southern lobe is visible in the optical and visual extinction has been shown to be significantly higher towards S106 IR and the northern lobe, Felli et al. 1984). We thus interpret the higher velocity blue and red [ $\mathrm{C}_{\mathrm{II}}$ ] emission $\left(v<-4\right.$ and $\left.v>1 \mathrm{~km} \mathrm{~s}^{-1}\right)$ as arising from swept-up material at the front and back sides of the expanding, wind driven hourglass. In this picture, one expects net blue shifted emission (with respect to the bulk molecular emission at $\sim-1 \mathrm{~km} \mathrm{~s}^{-1}$ ) from those parts of the hourglass close to S106 IR that are curved towards the observer (front side) and red shifted emission from those curved away (back side). Indeed, blue shifted $\mathrm{CO}$ and [ $\left.\mathrm{C}_{\mathrm{II}}\right]$ emission is particularly prominent in the northern and southern lobe close to S106 IR where the dark cone converges towards S106 IR in the foreground ( -4 to $-2 \mathrm{~km} \mathrm{~s}^{-1}$ velocity channels).

As most of the front wall along the main axis of the southern lobe has been eroded, we do not expect extreme blue shifted emission there. The backside of the southern lobe should also show blue emission because of its tilt towards the observer. We suggest that this emission is shifted towards the red due to the radial expansion of the lobe. Red shifted emission is observed only towards the southern lobe very close to S106 IR and quite faint further along its axis, which may indicate that the back side of the northern lobe has also been eroded.

For emission around the velocity of the bulk of the molecular gas, $\left[\mathrm{C}_{\mathrm{II}}\right]$ traces the side walls of the lobes (i.e., where the radial component of the velocity is smallest). Comparing [C II] and $\mathrm{CO} 11 \rightarrow 10$ emission to ${ }^{13} \mathrm{CO} 2 \rightarrow 1$ (Fig. A.1) allows to better characterize their relation to the bulk of the molecular cloud that is well traced in the low- $J$ CO line. [ $\mathrm{C}_{\mathrm{II}}$ ] (and to a certain degree $\mathrm{CO} 11 \rightarrow 10$ ) in the -3 to $+1 \mathrm{~km} \mathrm{~s}^{-1}$ range nicely traces the surfaces of the molecular cloud. We therefore attribute the $\left[\mathrm{C}_{\mathrm{II}}\right]$ emission in this velocity range as arising from PDRs in the cavity walls of the ionized lobes, directly correlated with the bulk emission of the molecular cloud. Further support for the above scenario comes from the overlays of [C $\mathrm{II}]$ velocity channels on continuum data from the VLA (emission from the $\mathrm{H}$ in region) near-IR from Subaru (emission of hot dust), and SHARCII (high columns of warm dust) in Fig. A.2. The comparison with the $350 \mu \mathrm{m}$ data is particularly interesting as it shows that [C $\mathrm{II}]$ is confined to or even funneled into the lobes by the high column density, warm dust seen in the submm continuum (the dark cone and S106 FIR). We even observe the dark lane sandwiched between $[\mathrm{C} \mathrm{II}]$ and ${ }^{13} \mathrm{CO} 2 \rightarrow 1$ emission, showing that there is a layering of warm ionized gas, warm dust, and colder molecular cloud material at the surface of the cloud. The strongest [C II] emission originates from the edge of S106 FIR, which is probably a signature of ablation or evaporation of this dense clump due to radiation and/or wind from the star.

Assuming optically thin, thermalized emission and beam filling of unity, we estimate lower limits to the beam averaged [C II] column density for the emission in the channel maps following (Crawford et al. 1985) in the high density, high temperature limit. For the [C II] wave length of $158 \mu \mathrm{m}$, an integrated intensity of $1 \mathrm{~K} \mathrm{~km} \mathrm{~s}^{-1}$ translates into $7.041 \times$ $10^{-6} \mathrm{erg} \mathrm{s}^{-1} \mathrm{~cm}^{-2} \mathrm{sr}^{-1}$ and a column density of $4.6 \times 10^{15} \mathrm{~cm}^{-2}$. The total $[\mathrm{C} \mathrm{II}]$ column density towards the emission peak is $2.0 \times 10^{18} \mathrm{~cm}^{-2}$, while the values for the channel maps in 


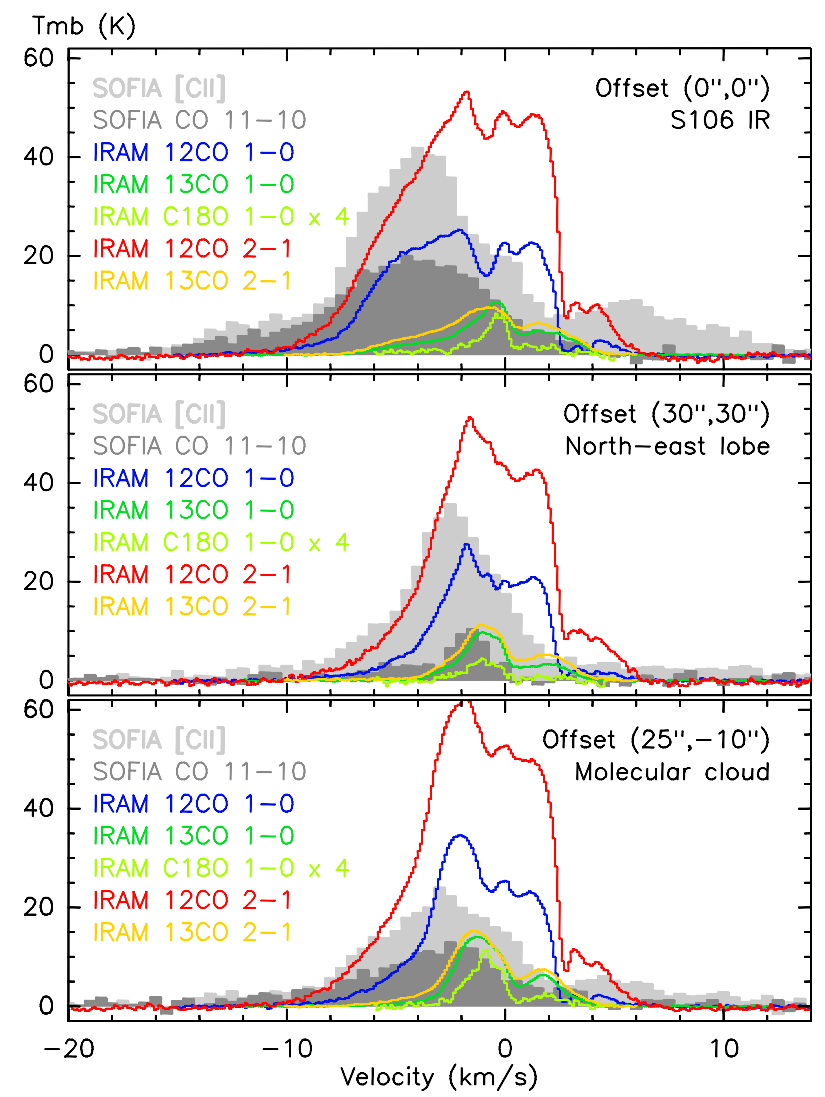

Fig. 2. [C II] and CO $11 \rightarrow 10$ spectra from SOFIA together with low$J$ CO lines from IRAM (Schneider et al. 2003) at a common angular resolution of 25". Offsets are given in arcsec from S106 IR. From top to bottom: S106 IR, north-east lobe, and molecular cloud.

$2 \mathrm{~km} \mathrm{~s}^{-1}$ bins span a small range from 1.4 to $2.4 \times 10^{17} \mathrm{~cm}^{-2}$, the maximum being in the $-4 \mathrm{~km} \mathrm{~s}^{-1}$ channel. The $[\mathrm{C}$ II] column density in the red velocity bins is on the order of $7.3 \times 10^{16} \mathrm{~cm}^{-2}$.

\subsection{Spectral signatures of high velocity gas}

The above findings are also reflected in the spectra displayed in Fig. 2 for three selected positions. The spectra have a common angular resolution of $25^{\prime \prime}$ and show line profiles towards S106 IR, the north-eastern lobe (cavity wall), and the molecular cloud. [C II] emission peaks at velocities of $\sim-4 \mathrm{~km} \mathrm{~s}^{-1}$, blue shifted from the peak velocity of the low- $J$ CO lines at $1 \mathrm{~km} \mathrm{~s}^{-1}$, and is generally very broad with emission covering the range from -14 to $+12 \mathrm{~km} \mathrm{~s}^{-1}$. This implies that [C II] traces very well the dynamics of warm gas in the PDR-layers, shocks, or the ionized phase, which most likely all have slightly different velocities and are subject to enhanced turbulence, quite different from the bulk of the molecular cloud.

The CO $11 \rightarrow 10$ line peaks near S106 IR at velocities around $-4 \mathrm{~km} \mathrm{~s}^{-1}$ and shows broad lines due to the stellar wind hitting the molecular gas, thus tracing the warmest part of the molecular cloud edge in the foreground closest to the star where the wind speed is higher or even shocks can play a role. At this position, we sample emission from both the $[\mathrm{C}$ II] and the $\mathrm{CO} 11 \rightarrow 10$ peak. Towards the north-eastern lobe, CO $11 \rightarrow 10$ becomes much weaker and the peak velocity shifts towards that of the lower- $J$ lines, likely reflecting that temperatures and densities are too low for sufficient excitation in the more quiescent gas outside the $\mathrm{H}$ II region. Low-J CO lines with high to moderate optical depth also show blue wing emission, but not as extended to the far blue side as observed for [C $\mathrm{II}$ ] and $\mathrm{CO} 11 \rightarrow 10$. This confirms that the latter lines trace gas in the foreground closer to the star and detached from the bulk of the colder molecular gas. High velocity red emission above $+6 \mathrm{~km} \mathrm{~s}^{-1}$ is only prominent in [C II] and not seen in any of the other tracers.

\section{Summary}

Our new spectrally resolved observations of [C II] and CO $11 \rightarrow 10$ reveal very complex morphology and kinematics of the warm gas in S106 with multiple velocity components at different locations relative to the exciting star, the dark lane, which we show to be composed of warm, high column density gas, and the bulk of the colder molecular gas. The [C II] and high- $J$ CO emitting material is highly dynamic with broad, nonGaussian wings in particular in [C II] showing no counterpart in any of the other observed tracers for the highest red and blue shifted velocities.

Only the high spectral and angular resolution provided by GREAT on SOFIA make it possible to disentangle emission components arising from the PDR surfaces at the inner cavity walls of the two $\mathrm{H}$ II region lobes, from swept-up gas due to the stellar wind of S106 IR and/or the expansion of the lobes, and from the $\mathrm{H}$ II region itself. To quantify how much of the emission is contributed by shocks, $\mathrm{H}$ II region, or PDR gas requires a more detailed analysis involving modeling, which will be addressed in a forthcoming paper.

Acknowledgements. Based in part on observations made with the NASA/DLR Stratospheric Observatory for Infrared Astronomy. SOFIA Science Mission Operations are conducted jointly by the Universities Space Research Association, Inc., under NASA contract NAS2-97001, and the Deutsches SOFIA Institut under DLR contract 50 OK 0901. We thank the SOFIA engineering and operations teams and the DSI telescope engineering team for their tireless support and good-spirit teamwork, which has been essential for the GREAT accomplishments during Early Science.

\section{References}

Bally, J., \& Scoville, N. Z. 1982, ApJ, 255, 497

Bally, J., Snell, R. L., \& Predmore, R. 1983, ApJ, 272, 154

Barsony, M., Scoville, N. Z., Bally, J., \& Claussen, M. J. 1989, ApJ, 343, 212

Crawford, M. K., Genzel, R., Townes, C. H., \& Watson, D. M. 1985, ApJ, 291, 755

Felli, M., Massi, M., Staude, H. J., et al. 1984, A\&A, 135, 261

Furuya, R. S., Kitamura, Y., Saito, M., et al. 1999, ApJ, 525, 821

Gibb, A. G., \& Hoare, M. G. 2007, MNRAS, 380, 246

Graf, U. U., Eckart, A., Genzel, R., et al. 1993, ApJ, 405, 249

Guan, X., Stutzki, J., Graf, U., et al. 2012, A\&A, 542, L4

Harris, A. I., Stutzki, J., Genzel, R., et al. 1987, ApJ, 322, L49

Heyminck, S., Graf, U. U., Güsten, R., et al. 2012, A\&A, 542, L1

Hoare, M. G., \& Muxlow, T. B. 1996, Radio Emission from the Stars and the Sun, 93, 47

Hodapp, K. W., \& Schneider, N. 2008, Handbook of Star Forming Regions, Vol. I, 90

Little, L. T., Kelly, M. L., Habing, R. J., \& Millar, T. J. 1995, MNRAS, 277, 307

Noel, B., Joblin, C., Maillard, J. P., \& Paumard, T. 2005, A\&A, 436, 569

Oasa, Y., Tamura, M., Nakajima, Y., et al. 2006, AJ, 131, 1608

Richer, J. S., Padman, R., Ward-Thompson, D., et al. 1993, MNRAS, 262, 839

Schneider, N., Simon, R., Kramer, C., et al. 2002, A\&A, 384, 225

Schneider, N., Simon, R., Kramer, C., et al. 2003, A\&A, 406, 915

Schneider, N., Simon, R., Bontemps, S., et al. 2007, A\&A, 474, 873

Simon, M., \& Fischer, J. 1982, BAAS, 14, 925

Smith, N., Jones, T. J., Gehrz, R. D., et al. 2001, AJ, 121, 984

Solf, J., \& Carsenty, U. 1982, A\&A, 116, 54

Stutzki, J., \& Winnewisser, G. 1985, A\&A, 144, 13

Stutzki, J., Ungerechts, H., \& Winnewisser, G. 1982, A\&A, 111, 201

Pages 5 to 6 are available in the electronic edition of the journal at http: //www . aanda.org 
R. Simon et al.: SOFIA observations of S106: dynamics of the warm gas

\section{Appendix A: Channel maps}

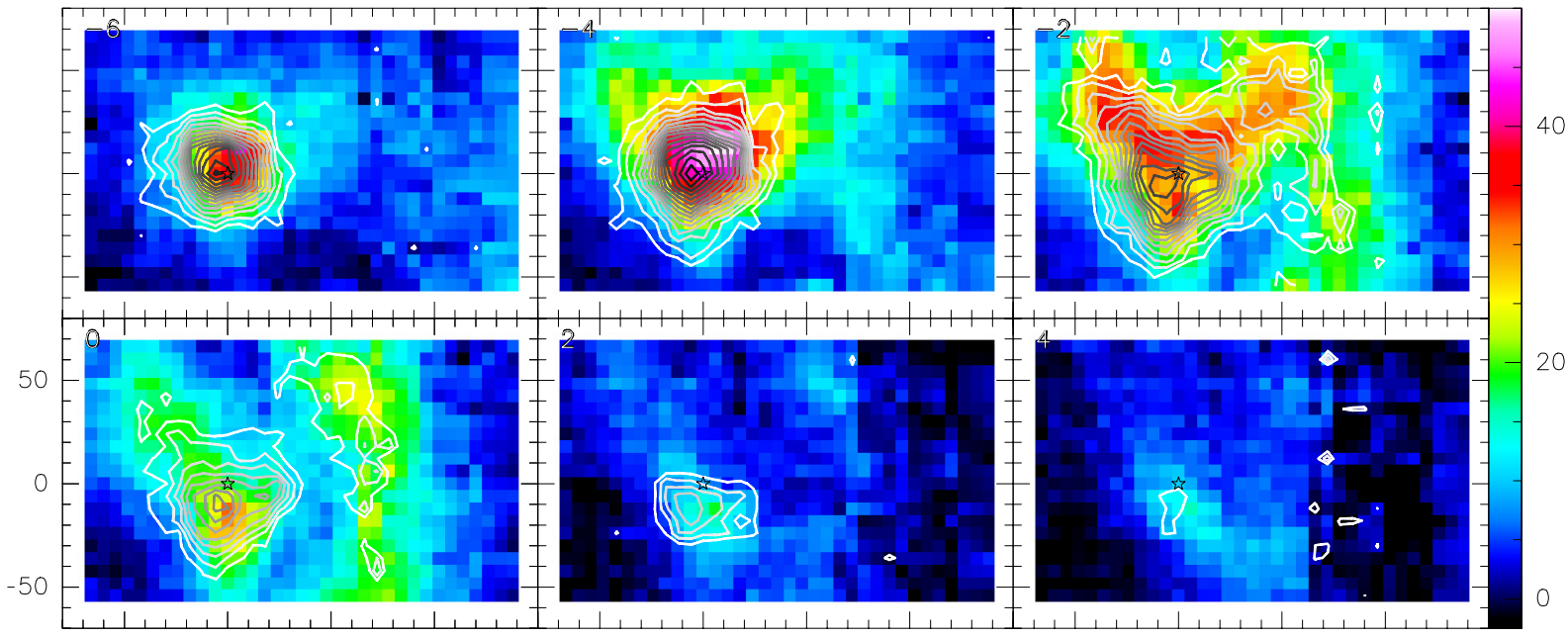

$50 \quad 0 \quad-50 \quad-100-150$
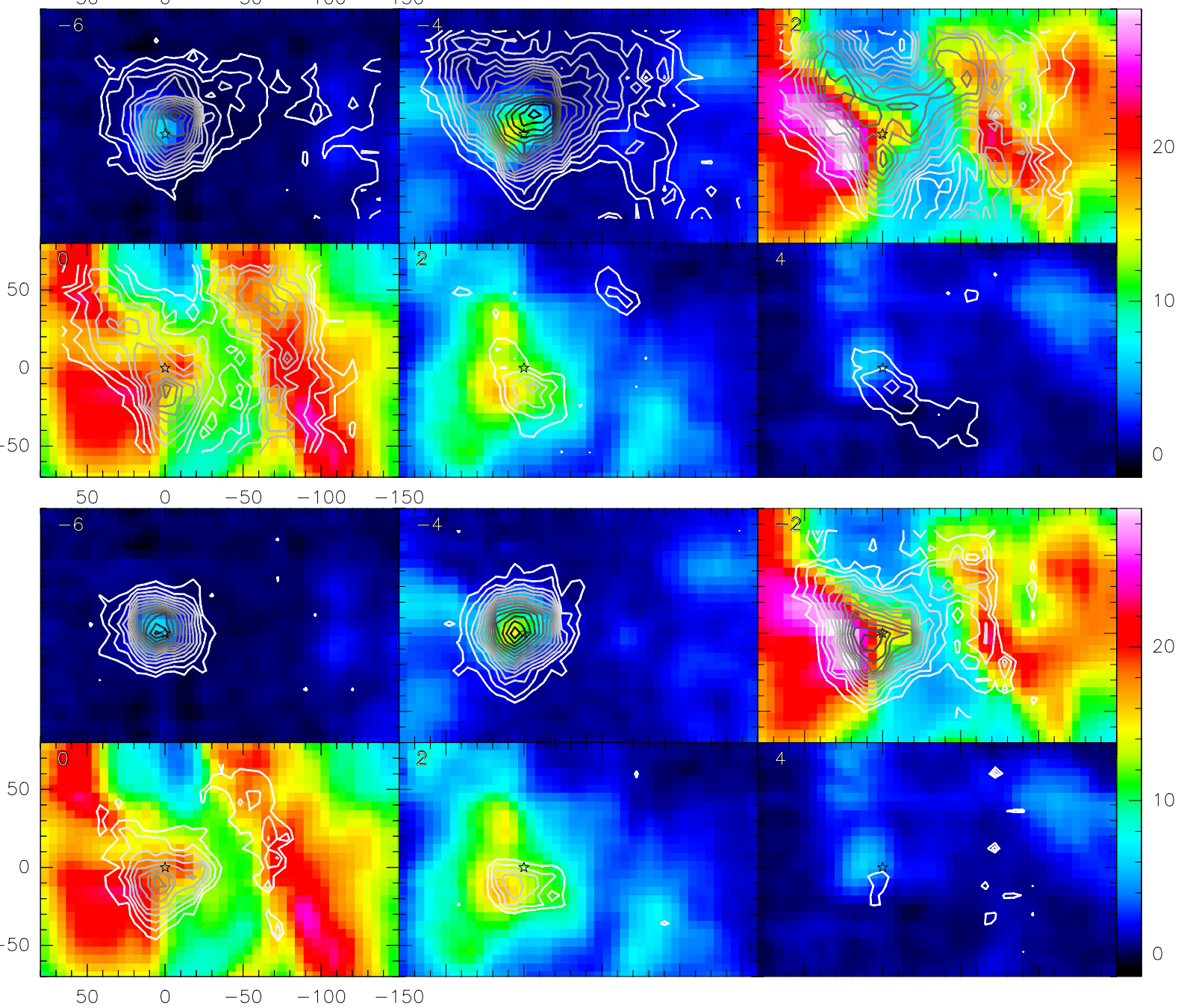

Fig. A.1. Channel maps of $\mathrm{CO} 11 \rightarrow 10$ contours on $[\mathrm{C} \mathrm{II}](t o p)$ and $[\mathrm{C} \mathrm{II}]$ and $\mathrm{CO} 11 \rightarrow 10$ contours on ${ }^{13} \mathrm{CO} 2 \rightarrow 1$ (middle and bottom). 

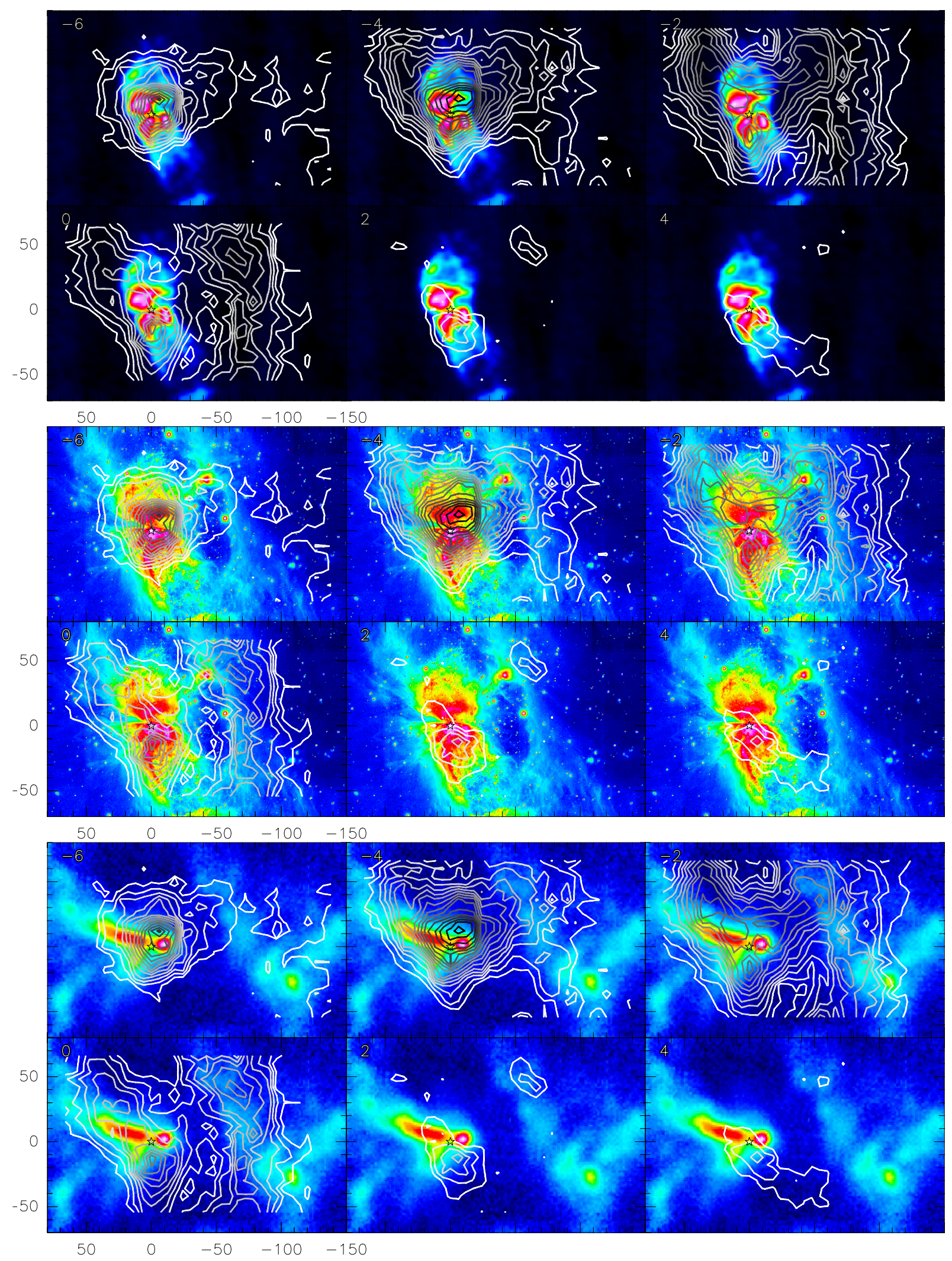

Fig. A.2. Top: channel maps of CII contours on a VLA $1.47 \mathrm{~cm}$ image. Middle: channel maps of CII contours on Subaru near-IR. Bottom: channel maps of CII contours on SHARC-II submm continuum. 